hep-ph/yymmddd

\title{
Gauge coupling and fermion mass relations in low string scale brane models
}

\author{
D.V. Gioutsos, G.K. Leontaris and J. Rizos \\ Theoretical Physics Division, Ioannina University, GR-45110 Ioannina, Greece
}

\begin{abstract}
We analyze the gauge coupling evolution in brane inspired models with $U(3) \times U(2) \times$ $U(1)^{N}$ symmetry at the string scale. We restrict to the case of brane configurations with two and three abelian factors $(N=2,3)$ and where only one Higgs doublet is coupled to down quarks and leptons and only one to the up quarks. We find that the correct hypercharge assignment of the Standard Model particles is reproduced for six viable models distinguished by different brane configurations. We investigate the third generation fermion mass relations and find that the correct low energy $m_{b} / m_{\tau}$ ratio can be obtained for $b-\tau$ Yukawa coupling equality at a string scale as low as $M_{S} \sim 10^{3} \mathrm{TeV}$.
\end{abstract}




\section{Introduction}

Low scale unification of gauge and gravitational interactions [1, 2, 3] appears to be a promising framework for solving the hierarchy problem. In this context, the weakness of the gravitational force in long distances is attributed to the existence of extra dimensions at the Fermi scale. A realization of this scenario can occur in type I string theory [4] where gauge interactions are mediated by open strings with their ends attached on some D-brane stack, while gravity is mediated by closed strings that propagate in the whole 10-dimensional space.

In the context of Type I string theory using appropriate collections of parallel ${ }^{1}$ or intersecting [7, 8] D-branes, there has been considerable work in trying to derive the Standard Model theory or its Grand Unified extensions [9]-[19]. Some of these low energy models revealed rather interesting features: (i) The correct value of the weak mixing angle can be reproduced for a string scale of the order of a few $\mathrm{TeV}$ (ii) baryon and lepton numbers are conserved due to the existence of exact global symmetries which are remnants of additional anomalous $U(1)$ factors broken by the Green-Schwarz mechanism (iii) supersymmetry is not necessary to solve the hierarchy problem.

However, its rivals, supersymmetric Grand Unified theories and their heterotic string realizations exhibit additional interesting features as: (i) full gauge coupling unification, that occurs to a scale of the order $10^{16} \mathrm{GeV}$ or a little higher when additional matter thresholds are introduced (see eg. [20]) (ii) fermion mass relations [21, 22] and in particular bottom-tau unification, i.e., the equality of the corresponding Yukawa couplings at the unification scale, which reproduces the correct mass relation $m_{b} / m_{\tau}$ at low energies.

In Type I string scenarios the volume of the internal space enters in the relation between gauge and string couplings 23. and in general predictability is lost. However, there exist classes of models where (due to some internal volume relation in intersecting brane models or superposition of the associated parallel brane sets) at least the two of the brane couplings are either related or equal at the string scale $M_{S}$ (e.g., "petite unification" 15, 24]). For models with three gauge group factors one such relation is enough to associate the low energy data, i.e., the Weinberg angle and the strong coupling, with the string scale. Given the variety of the type I vacua one could follow a bottom-up approach and consider ratios of brane couplings as free parameters. In this context, some restrictions on the parameter space can be obtained assuming certain fermion mass relations at the string scale. We will concentrate here on restrictions implied by the heaviest generation fermion mass relations and in particular the bottom - tau Yukawa couplings at the string scale.

Following a bottom-up approach, in this letter we examine the possible brane configurations that can accommodate the Standard Model and the associated hypercharge embeddings and we analyze the consequences of (partial) gauge coupling unification in conjunction with bottom-tau coupling equality. We shall restrict to non-supersymmetric configurations, (for some recent results on supersymmetric models see [17]), however, we will consider models with two Higgs doublets so that the bottom and top quark masses

\footnotetext{
${ }^{1}$ For reviews see [5], [6].
} 
will be related to different vacuum expectation values while the tau lepton and the bottom quark will receive masses from the same Higgs doublet. We find that in a class of models that can be realized in the context of type I string theory with large extra dimensions, the experimentally low energy masses can be reproduced assuming equality of bottom-tau Yukawa couplings and a string scale as low as $M_{S} \sim 10^{3} \mathrm{TeV}$.

In the next section we briefly describe the general set up of brane models and identify candidate brane configurations that allow bottom-tau coupling equality. All possible hypercharge embeddings in the presence of additional $U(1)$ factors are classified. Section 3 deals with the calculational details and renormalization analysis of gauge and Yukawa couplings, while in section 4 the results for $b-\tau$ couplings are presented. Our conclusions are drawn in section 5 .

\section{Hypercharge embedding in generic Standard model like brane configurations}

In this work, we consider models which arise in the context of various D-brane configurations 9, 10]. A single D-brane carries a $U(1)$ gauge symmetry which is the result of the reduction of the ten-dimensional Yang-Mills theory. Therefore, a stack of $n$ parallel, almost coincident D-branes gives rise to a $U(n)$ gauge theory where the gauge bosons correspond to open strings having both their ends attached to some of the branes of the various stacks.

The minimal number of brane sets required to provide the Standard Model structure is three: a 3-brane "color" stack with gauge symmetry $U(3)_{C} \sim S U(3)_{C} \times U(1)$ gives rise to strong interactions, a 2-brane "weak" stack gives rise to $U(2)_{L} \sim S U(2)_{L} \times U(1)$ gauge symmetry that includes the weak interactions and an abelian $U(1)$ brane for hypercharge. However, accommodation of all SM particles as open strings between different brane sets requires at least one $U(1)$ brane to be added to the above configuration [9, 14. Additional abelian branes may be present too. In more complicated scenarios the weak or color stacks can be repeated leading to an effective "higher level embedding" of the Standard Model. The full gauge group will be of the form

$$
G=U(m)_{C}^{p} \times U(n)_{L}^{q} \times U(1)^{N}
$$

with $m \geq 3$ and $n \geq 2$ and $p, q \geq 1$. Since $U(n) \sim S U(n) \times U(1)$ and so on, we infer that brane constructions automatically give rise to models with $S U(n)$ gauge group structure and several $U(1)$ factors.

A generic feature of this type of string vacua is that several abelian gauge factors are anomalous. Note that this is in contrast to the heterotic case where at most one $U(1)$ is anomalous, however, anomalies are cancelled by a generalized Green-Schwarz mechanism. At least one $U(1)$ combination remains anomaly free. This is the hypercharge that can be in general written as

$$
Y=\sum_{i=1}^{p} k_{3}^{(i)} Q_{3}^{i}+\sum_{j=1}^{q} k_{2}^{(j)} Q_{2}^{j}+\sum_{\ell=1}^{N} k_{\ell}^{\prime} Q_{\ell}^{\prime}
$$


where $Q_{3}^{i}$ are the $U(1)$ generators of the color factor $i, Q_{2}^{j}$ are the $U(1)$ generators of the weak factor $j$ and $Q_{\ell}^{\prime}(\ell=1, \ldots, N)$, are the generators of the remaining Abelian factors.

The simplest case which leads directly to the SM theory is the choice $p=q=1$. Constructions of this type have already been proposed in the papers of reference [9]. An immediate consequence of (11) and (2) is that the hypercharge coupling $\left(g_{Y}\right)$ at the string/brane scale $\left(M_{S}\right)$ is related to the brane couplings $\left(g_{m}, g_{n}, g_{i}^{\prime}\right)$ as $^{2}$

$$
\frac{1}{g_{Y}^{2}}=\frac{2 m k_{3}^{2}}{g_{m}^{2}}+\frac{2 n k_{2}^{2}}{g_{n}^{2}}+2 \sum_{i=1}^{N} \frac{{k_{i}^{\prime}}^{2}}{{g_{i}^{\prime}}^{2}}
$$

where we have used the traditional normalization $\operatorname{Tr} T^{a} T^{b}=\delta^{a b} / 2, a, b=1, \ldots, n^{2}$ for the $U(n)$ generators and assumed that the vector representation $(\mathbf{n})$ has abelian charge +1 and thus the $U(1)$ coupling becomes $g_{n} / \sqrt{2 n}$ where $g_{n}$ the $S U(n)$ coupling.

Choosing further $m=3, n=2$ in (3) we obtain directly the non-abelian structure of the SM with several $U(1)$ factors, therefore the hypercharge gauge coupling condition reads

$$
\frac{1}{g_{Y}^{2}}=\frac{6 k_{3}^{2}}{g_{3}^{2}}+\frac{4 k_{2}^{2}}{g_{2}^{2}}+2 \sum_{i=1}^{N} \frac{{k_{i}^{\prime}}^{2}}{g_{i}^{\prime 2}}
$$

For a given hypercharge embedding the $k_{i}^{\prime}$ 's are known and equation (44) relates the weak angle with the gauge coupling ratios at the string scale

$$
\begin{aligned}
\sin ^{2} \theta_{W}\left(M_{S}\right) & =\frac{1}{1+k_{Y}} \\
k_{Y} & \equiv \frac{\alpha_{2}}{\alpha_{Y}}=6 k_{3}^{2} \frac{\alpha_{2}}{\alpha_{3}}+4 k_{2}^{2}+2 \sum_{i=1}^{N}{k_{i}^{\prime}}^{2} \frac{\alpha_{2}}{\alpha_{i}^{\prime}}
\end{aligned}
$$

where $\alpha_{i} \equiv g_{i}^{2} /(4 \pi)$.

Given a relation between the $\alpha_{i}^{\prime}$ and $\alpha_{2}$ (or $\alpha_{3}$ ), equations (56) in conjunction with the $\alpha_{3}$ evolution equation, determine the string scale $M_{S}$. In the remaining of this section, we will derive all possible sets of $k_{i}$ 's compatible with brane configurations which embed the SM particles and imply an economical Higgs spectrum.

In brane models each SM particle corresponds to an open string stretched between two branes. In our charge conventions, the possible quantum numbers of such a string ending to the $U(m)$ and $U(n)$ brane sets are $(\mathbf{m} ;+1, \mathbf{n} ;+1),(\overline{\mathbf{m}} ;-1, \mathbf{n} ;+1),(\mathbf{m} ;+1, \overline{\mathbf{n}} ;-1)$, $(\overline{\mathbf{m}} ;-1, \overline{\mathbf{n}} ;-1)$, that is, bi-fundamentals of the associated unitary groups. Higher antisymmetric or symmetric representations could also be obtained by considering strings with both ends on the same brane set, however, we will restrict here to the bi-fundamental case. In order to ensure $\lambda_{b}-\lambda_{\tau}$ unification at $M_{S}$, we assume models where down quarks and leptons acquire their masses from a common Higgs. Only two configurations $(N=2,3)$ share the above properties and are presented pictorially in Fig. 1].

\footnotetext{
${ }^{2}$ If some Cartan generators of $S U(m)$ also contribute to the hypercharge, the formula becomes model dependent, see e.g. [15.
} 

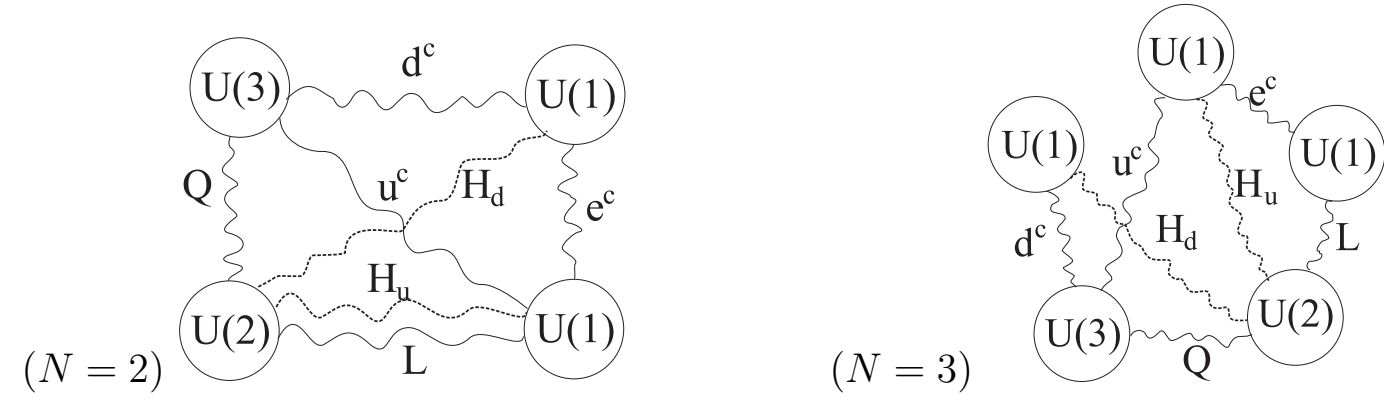

Figure 1: Possible $N=2,3$ brane configurations ( $N$ is the number of the $U(1)$-branes) that can accommodate the SM spectrum with down quarks and leptons acquiring masses from the same Higgs doublet. The first consists of four brane sets and has gauge symmetry $U(3) \times U(2) \times U(1)^{2}$ and the second consists of five brane sets and has gauge group $U(3) \times$ $U(2) \times U(1)^{3}$.

The possible hypercharge embeddings for each configuration can be obtained by solving the hypercharge assignment conditions for SM particles. Generically, the SM particle abelian charges under $U_{3}(1) \times U_{2}(1) \times U(1)^{\prime}{ }_{1} \times U(1)^{\prime}{ }_{2}$ are of the form $Q\left(+1, \epsilon_{1}, 0,0\right)$, $d^{c}\left(-1,0, \epsilon_{2}, 0\right), u^{c}\left(-1,0,0, \epsilon_{3}\right), L\left(0, \epsilon_{4}, 0, \epsilon_{5}\right), e^{c}\left(0,0, \epsilon_{6}, \epsilon_{7}\right)$, where $\epsilon_{i}= \pm 1, i=1, \ldots, 7$. Then, the SM hypercharge assignment conditions read

$$
\begin{aligned}
k_{3}+k_{2} \epsilon_{1} & =\frac{1}{6} \\
-k_{3}+k_{1}^{\prime} \epsilon_{2} & =\frac{1}{3} \\
-k_{3}+k \epsilon_{3} & =-\frac{2}{3} \\
k_{2} \epsilon_{4}+k_{2}^{\prime} \epsilon_{5} & =-\frac{1}{2} \\
k_{1}^{\prime} \epsilon_{6}+k_{2}^{\prime} \epsilon_{7} & =1
\end{aligned}
$$

for $Q, d^{c}, u^{c}, L$ and $e^{c}$ respectively. Here we have used a compact notation where $k=k_{2}^{\prime}$ for the first configuration and $k=k_{3}^{\prime}$ for the second one. ${ }^{3}$

As seen by (2) and (31), only the absolute values of the hypercharge embedding coefficients $k_{i}, k_{i}^{\prime}$ enter the coupling relation at $M_{S}$. Solving (7), for the SM particle charges in configuration $(N=2)$ we obtain three possible solutions. These correspond to the (absolute) values for the coefficients presented in cases (a),(b) and (c) of Table 1. Configuration $N=3$ leads to four additional cases, namely (d), (e),(f) and (g) of the same Table. If in a particular solution a coefficient $k_{i}$ (or $k_{i}^{\prime}$ ) turns out to be zero, the associated abelian factor does not participate to the hypercharge.

\footnotetext{
${ }^{3}$ In some of our solutions there exist additional unbroken $U(1)$ 's factors. We will assume in the sequel that these $U(1)$ 's will be broken by vacuum expectation values of additional SM singlet Higgs fields or at
} 


\begin{tabular}{|c|c|c|c|c|c|c|}
\hline$N$ & & $\left|k_{3}\right|$ & $\left|k_{2}\right|$ & $\left|k_{1}^{\prime}\right|$ & $\left|k_{2}^{\prime}\right|$ & $\left|k_{3}^{\prime}\right|$ \\
\hline \multirow{4}{*}{2} & $(\mathrm{a})$ & $\frac{1}{6}$ & 0 & $\frac{1}{2}$ & $\frac{1}{2}$ & - \\
& $(\mathrm{b})$ & $\frac{2}{3}$ & $\frac{1}{2}$ & 1 & 0 & - \\
& $(\mathrm{c})$ & $\frac{1}{3}$ & $\frac{1}{2}$ & 0 & 1 & - \\
\hline \multirow{5}{*}{3} & $(\mathrm{~d})$ & $\frac{1}{6}$ & 0 & $\frac{1}{2}$ & $\frac{1}{2}$ & $\frac{1}{2}$ \\
& $(\mathrm{e})$ & $\frac{1}{3}$ & $\frac{1}{2}$ & 0 & 1 & 1 \\
& $(\mathrm{f})$ & $\frac{5}{6}$ & 1 & $\frac{1}{2}$ & $\frac{1}{2}$ & $\frac{3}{2}$ \\
& $(\mathrm{~g})$ & $\frac{2}{3}$ & $\frac{1}{2}$ & 1 & 0 & 0 \\
\hline
\end{tabular}

Table 1: Absolute values of the possible hypercharge embedding coefficient sets $\left(k_{3}, k_{2}\right.$ and $k_{i}^{\prime}$ ) for the brane configurations with $N=2$ and $N=3$ of Figure 1

\section{Gauge coupling running and the String scale}

As already mentioned, in the low energy Type I string scenarios the gauge couplings do not unify at the string scale $M_{S} .{ }^{4}$ However, it has been observed that in some cases low energy data are compatible with a partially unified model where some of the gauge couplings are equal ("petite unification" [15, 24]). At the string level this scenario corresponds to superposing the associated parallel brane stacks. Moreover certain coupling relations arise in classes of intersecting brane models. Given the fact that there may exist various gauge coupling relations at the string scale $M_{S}$ (although, only one for a minimal model), low energy electroweak data can be used to determine $M_{S}$ through the renormalization group equations (RGEs). Following this bottom-up approach, in this section we determine the range of the string scale for all the above models by taking into account the experimental values of $\alpha_{3}, \alpha_{e m}$ and $\sin ^{2} \theta_{W}$ at $M_{Z}$ [25]

$$
\alpha_{3}=0.118 \pm 0.003, \quad \alpha_{e}^{-1}=127.906, \quad \sin ^{2} \theta_{W}=0.23120
$$

For the scales above $M_{Z}$ we consider the standard model spectrum with two Higgs doublets. The one loop RGEs for the gauge couplings $(\tilde{\alpha} \equiv \alpha /(4 \pi))$ take the form

$$
\frac{d \tilde{\alpha}_{i}}{d t}=b_{i} \tilde{\alpha}_{i}^{2}, \quad i=Y, 2,3
$$

where $\left(b_{Y}, b_{2}, b_{3}\right)=(7,-3,-7)$ and $t=2 \ln \mu$ ( $\mu$ is the renormalization point).

First, we concentrate on simple relations of the gauge couplings, i.e., those relations implied from models arising only in the context on non-intersecting branes. In these cases, certain constraints on the initial values of the gauge couplings have to be taken into account,

the string level by six-dimensional anomalies [14].

${ }^{4}$ One may think that the predictability of these constructions is lost, however, bearing in mind that the origin of each gauge factor is due to a different stack of branes, the situation of unequal couplings at the effective string scale, although not predictive, looks completely natural. 


\begin{tabular}{|c|c|c|c||c|c|c|}
\hline & \multicolumn{6}{|c|}{ Model } \\
\hline $\begin{array}{c}\text { coupling } \\
\text { relation }\end{array}$ & $(\mathrm{a})$ & $(\mathrm{b})$ & $(\mathrm{c})$ & $(\mathrm{d})$ & $(\mathrm{e})$ & $(\mathrm{g})$ \\
\hline$\alpha_{i}^{\prime}=\alpha_{2}$ & $\frac{\xi}{6}+1$ & $\frac{8 \xi}{3}+3$ & $\frac{2 \xi}{3}+3$ & $\frac{\xi}{6}+\frac{3}{2}$ & $\frac{2 \xi}{3}+5$ & $\frac{8 \xi}{3}+3$ \\
\hline$\alpha_{i}^{\prime}=\alpha_{3}$ & $\frac{7 \xi}{6}$ & $\frac{14 \xi}{3}+1$ & $\frac{8 \xi}{3}+1$ & $\frac{2 \xi}{3}+1$ & $\frac{14 \xi}{3}+1$ & $\frac{14 \xi}{3}+1$ \\
\hline$($ see text) & $\frac{2 \xi}{3}+\frac{1}{2}$ & - & - & $\frac{5 \xi}{3}$ & $\frac{8 \xi}{3}+3$ & - \\
\hline$($ see text) & - & - & - & $\frac{7 \xi}{6}+\frac{1}{2}$ & - & - \\
\hline \hline$M_{S}(\mathrm{GeV})$ & $6.71 \times 10^{17}$ & $5.78 \times 10^{3}$ & $1.99 \times 10^{6}$ & $1.65 \times 10^{14}$ & $5.78 \times 10^{3}$ & $5.78 \times 10^{3}$ \\
\hline
\end{tabular}

Table 2: The possible values of $k_{Y}$ as a function of $\xi=\frac{\alpha_{2}}{\alpha_{3}}$ for various orientations of $U(1)$ 's for the models of Table 1. The first row presents the $k_{Y}$ values when all $U(1)$ branes are aligned with the $S U(2)$ brane, i.e., when $\alpha_{i}^{\prime}=\alpha_{2}$, while the second one corresponds to $\alpha_{i}^{\prime}=\alpha_{3}$. The next two rows show the $k_{Y}$ values for other possible orientations (see text for details). Last row shows the minimum value of the string scale $M_{S}$ obtained for the models $a-g$.

leading to a discrete number of admissible cases which we are going to discuss. Thus, in the case of two $D 5$ branes, $U(3)$ and $U(2)$ are confined in different bulk directions. In the parallel brane scenario the orientation of a number of the extra $U(1)$ 's may coincide with the $U(3)$-stack direction while the remaining abelian branes are parallel to the $U(2)$ stack. This implies that the corresponding $U(1)$ gauge couplings have the same initial values either with the $\alpha_{3}$ or with the $\alpha_{2}$ gauge couplings. If we define $\xi=\frac{\alpha_{2}}{\alpha_{3}}$ the ratio of the two non-abelian gauge couplings at the string scale, for any distinct case, $k_{Y}$ takes the form $k_{Y}=\lambda \xi+\nu$, where $\lambda, \nu$ are calculable coefficients which depend on the specific orientation of the $U(1)$ branes. For example, in model (a) we can have the following possibilities: $\alpha_{1}^{\prime}=\alpha_{2}^{\prime}=\alpha_{2}, \alpha_{1}^{\prime}=\alpha_{2}^{\prime}=\alpha_{3}$ and $\alpha_{1}^{\prime}=\alpha_{2}, \alpha_{2}^{\prime}=\alpha_{3}$ leading to $k_{Y}=\frac{\xi}{6}+1, \frac{7 \xi}{6}$ and $\frac{2 \xi}{3}+\frac{1}{2}$ correspondingly. All cases for the models (a-g) are presented in Table 2 and are classified with regard to the hypercharge coefficient $k_{Y}$. (The analysis shows that all cases of Model (f) lead to unacceptably small string scales, so these are not presented). Allowing $\alpha_{3}$ to take values different from $\alpha_{2}$, we find that models (a,b,c,d,e,g) of Table 1 predict a string scale in a wide range, from a few $\mathrm{TeV}$ up to the Planck mass. The highest value is of the order $M_{S} \sim 7 \times 10^{17} \mathrm{GeV}$ and corresponds to equal couplings $\frac{\alpha_{2}}{\alpha_{3}} \equiv \xi=1$ at $M_{S}$. On the other hand, lower unification values of the order of a few $\mathrm{TeV}$ assume a gauge coupling ratio $\frac{\alpha_{3}}{\alpha_{2}} \approx 2$. In this case the idea of complete gauge coupling unification could be still valid, considering that the SM gauge group arises from the breaking of a gauge symmetry whose non-abelian part is $U(3) \times U(2)^{2}$, i.e., for the case $p=1, q=2$ of (11) where the factor of 2 in the gauge coupling ratio is related to the diagonal breaking $U(2) \times U(2) \rightarrow U(2)$. The distinct cases with the predictions for the unification scale and other quantities we are interested in, are presented in the columns (2-4) of Table 3. The lowest possible unification for the three models (b),(e),(g) corresponds to $k_{Y}=\frac{14 \xi}{3}+1$, and is $M_{S} \sim 5.81 \times 10^{3} \mathrm{GeV}$, for a weak to strong gauge coupling ratio $\xi \sim 0.42$ at $M_{S}$. Case (c) predicts an intermediate value $M_{S}=2 \times 10^{6} \mathrm{GeV}$ while model (d) gives $M_{S} \sim 10^{14} \mathrm{GeV}$. Finally, model (a) for 
$\xi \sim 1$ predicts a unification scale as high as $M_{S} \sim 6.7 \times 10^{17} \mathrm{GeV}$ which is of the order of the heterotic string scale. Interestingly, in this latter case, all gauge couplings are equal at $M_{S}, \alpha_{3}=\alpha_{2}=\alpha_{i}^{\prime}$, while, as can be seen from Table $2, k_{Y}$ takes a common value for all three cases, $k_{Y}=7 / 6$.

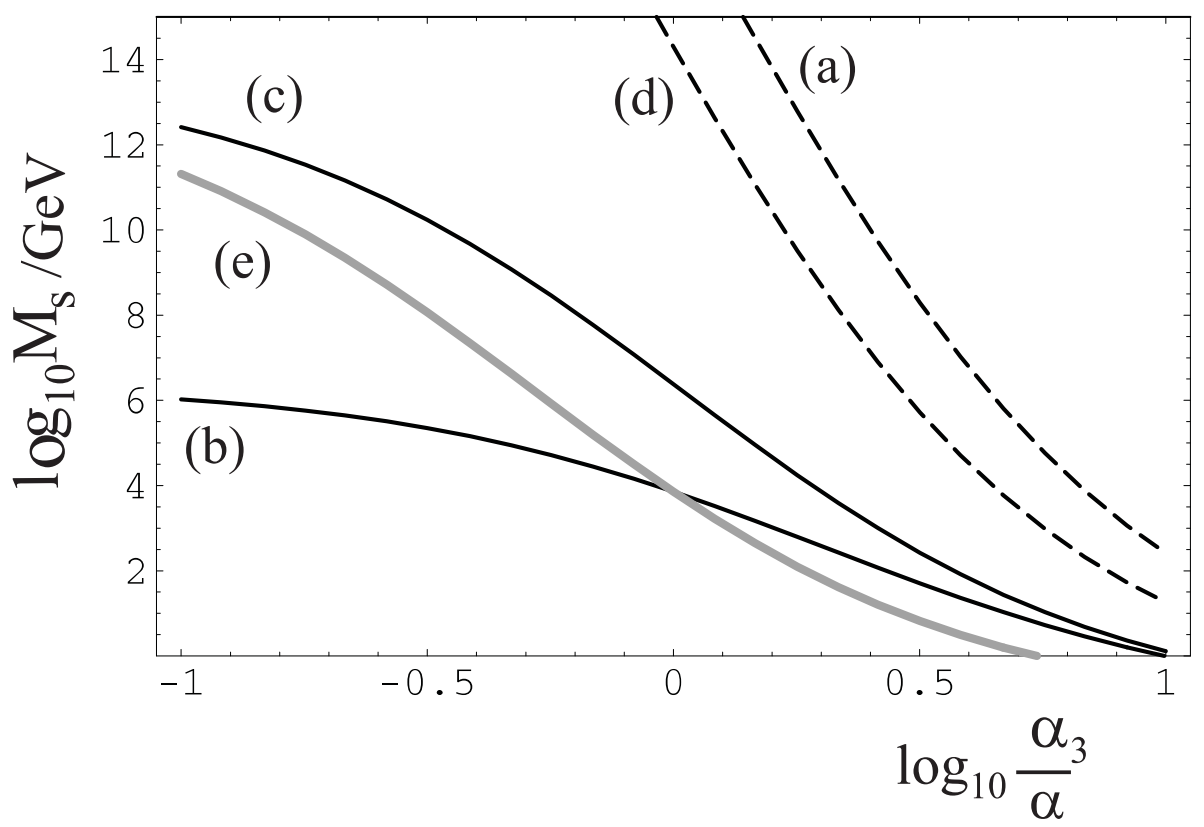

Figure 2: The string scale as a function of the coupling ratio $\frac{\alpha_{3}}{\alpha},(\alpha$ is a common value for the $U(1)$ couplings $\alpha_{i}^{\prime}$ ) for the different hypercharge embeddings of Table 1 , in the general case of intersecting branes. The results for model (g) are identical with those of model (b).

In the general intersecting case, the $U(1)$ branes are neither aligned to the $S U(3)$, nor to the $S U(2)$ stacks, thus the corresponding gauge couplings can take arbitrary values. Without loss of generality, we will assume here for simplicity that all these couplings are equal $\alpha_{1}^{\prime}=\alpha_{2}^{\prime}=\ldots=\alpha_{N}^{\prime}=\alpha$. In Figure 2 we plot the string scale $\left(M_{S}\right)$ as a function of the logarithm of the ratio $\alpha_{3} / \alpha$ for the candidate models (a), (b), (c), (d), (e) and (g). The results for models (b), (c), (e) and (g) which is identical with model (b), are represented in the figure with continuous lines. These are compatible with low scale unification particularly when $\alpha_{3} \geq \alpha$. For $\alpha_{i}^{\prime}=\alpha_{3}$, (which corresponds to the zero of the logarithm at the $x$-axis), we obtain again the results of the parallel brane scenario, shown in Table 2 . At this point, we further observe a crossing of the (e)-curve with the curve for models (b), (g). It is exactly this point $\left(\alpha_{i}^{\prime}=\alpha_{3}\right)$ that these three models predict the same value for the lowest string scale. When $\alpha_{3} \geq \alpha_{i}^{\prime}$, model (e) predicts the lowest $M_{S}$, whilst, if $\alpha_{i}^{\prime}>\alpha_{3}$, models (b), (g) imply lower string scales than model (e).

The values of the string scale for models (a), (d) (represented in the figure with dashed curves) are substantially higher; for these latter cases in particular, assuming reasonable gauge coupling relations $\alpha_{i}^{\prime} \approx \mathcal{O}\left(\alpha_{2,3}\right)$ we find that $M_{S} \geq 10^{12} \mathrm{GeV}$. Again, for $\alpha_{3}=\alpha_{i}^{\prime}$, (the zero value of the $x$-axis) we rederive the values of $M_{S}$ presented in Table 2. 


\section{Yukawa coupling evolution and mass relations}

In this section, we will examine whether a unification of the $b-\tau$ Yukawa couplings is possible in the above described low string scale models ${ }^{5}$. Our procedure is the following:

Using the experimentally determined values for the third generation fermion masses $m_{b}, m_{\tau}$ we run the 2-loop system of the $S U(3)_{C} \times U(1)_{Y}$ renormalization group equations up to the weak scale $\left(M_{Z}\right)$ and reconcile there the results with the experimentally known values for the weak mixing angle and the gauge couplings. For the renormalization group running below $M_{Z}$ we define the parameters

$$
\tilde{\alpha}_{e}=\left(\frac{e}{4 \pi}\right)^{2}, \tilde{\alpha}_{3}=\left(\frac{g_{3}}{4 \pi}\right)^{2}, t=2 \ln \mu
$$

where $e, g_{3}$ are the electromagnetic and strong couplings respectively and $\mu$ is the renormalization scale. The relevant RGEs are [27]

$$
\begin{aligned}
& \frac{d \tilde{\alpha}_{e}}{d t}=\frac{80}{9} \tilde{\alpha}_{e}^{2}+\frac{464}{27} \tilde{\alpha}_{e}^{3}+\frac{176}{9} \tilde{\alpha}_{e}^{2} \tilde{\alpha}_{3} \\
& \frac{d \tilde{\alpha}_{3}}{d t}=-\frac{23}{3} \tilde{\alpha}_{3}^{2}-\frac{116}{3} \tilde{\alpha}_{3}^{3}+\frac{22}{9} \tilde{\alpha}_{3}^{2} \tilde{\alpha}_{e}-\frac{9769}{54} \tilde{\alpha}_{3}^{4} \\
& \frac{d m_{b}}{d t}=m_{b}\left\{-\frac{1}{3} \tilde{\alpha}_{e}-4 \tilde{\alpha}_{3}+\frac{397}{162} \tilde{\alpha}_{e}^{2}-\frac{1012}{18} \tilde{\alpha}_{3}^{2}-\frac{4}{9} \tilde{\alpha}_{3} \tilde{\alpha}_{e}-474.8712 \tilde{\alpha}_{3}^{3}\right\} \\
& \frac{d m_{\tau}}{d t}=m_{\tau}\left\{-3 \tilde{\alpha}_{e}+\frac{373}{18} \tilde{\alpha}_{e}^{2}\right\}
\end{aligned}
$$

where $m_{b}, m_{\tau}$ are the running masses of the bottom quark and the tau lepton respectively, while we use the notation $\tilde{a}_{i} \equiv g_{i}^{2} / 16 \pi^{2}$ and $\tilde{a}_{t, b, \tau} \equiv \lambda_{t, b, \tau}^{2} / 16 \pi^{2}$.

The required value for the running mass of $m_{t}$ at $M_{Z}$ is computed as follows: we formally solve the 1-loop RGE system for $\left(\tilde{a}_{3}, \tilde{a}_{2}, \tilde{a}_{Y}, \tilde{a}_{t}, \tilde{a}_{b}, \tilde{a}_{\tau}\right)$ and afterwards we determine the interpolating function for $\tilde{a}_{3}(\mu)$ and $m_{t}\left(\mu ; m_{t}\left(M_{Z}\right)\right)$ at any scale $\mu$ above $M_{Z}$, where $m_{t}\left(M_{Z}\right)$ indicates the dependence on an arbitrary initial condition. The unknown value for $m_{t}\left(M_{Z}\right)$ is determined by solving numerically the algebraic equation

$$
\left[m_{t}\left(\mu ; m_{t}\left(M_{Z}\right)\right)-\frac{M_{t}}{1+\frac{16}{3} \tilde{a}_{3}(\mu)-2 \tilde{a}_{t}(\mu)}\right]_{\mu=M_{t}}=0
$$

We use these results as inputs for the relevant parameters and we run the RGE system to the scale where the $\tilde{a}_{b}$ and $\tilde{a}_{\tau}$ Yukawa couplings coincide. In our numerical analysis we use for the gauge couplings the values presented in the previous section, for the bottom quark mass $m_{b}$ the experimentally determined range at the scale $\mu=m_{b}$, i.e., $m_{b}\left(m_{b}\right)=4.25 \pm 0.15$ $\mathrm{GeV}$ and finally the top pole mass is taken to be $M_{t}=178.0 \pm 4.3 \mathrm{GeV}[25]$.

For the scales above $M_{Z}$ we consider the standard model spectrum augmented by one more Higgs. The Higgs doubling is in accordance with the situation that usually arises in

\footnotetext{
${ }^{5}$ For $b-\tau$ unification in a different context see also [26].
} 
the SM variants with brane origin. Moreover, we assume that one Higgs $H_{u}$ only couples to the top quark while the second Higgs $H_{d}$ couples only to the bottom. Then, in analogy with supersymmetry we define the angle $\beta$ related to their vevs where $\tan \beta=\frac{v_{u}}{v_{d}}$. Thus, we have the equations for the gauge couplings

$$
\frac{d \tilde{\alpha}_{Y}}{d t}=7 \tilde{\alpha}_{Y}^{2}, \frac{d \tilde{\alpha}_{2}}{d t}=-3 \tilde{\alpha}_{2}^{2}, \frac{d \tilde{\alpha}_{3}}{d t}=-7 \tilde{\alpha}_{3}^{2}
$$

and for the Yukawas

$$
\begin{aligned}
\frac{d \tilde{\alpha}_{t}}{d t} & =\tilde{\alpha}_{t}\left(-\frac{17}{12} \tilde{\alpha}_{Y}-\frac{9}{4} \tilde{\alpha}_{2}-8 \tilde{\alpha}_{3}+\frac{9}{2} \tilde{\alpha}_{t}+\frac{1}{2} \tilde{\alpha}_{b}\right) \\
\frac{d \tilde{\alpha}_{b}}{d t} & =\tilde{\alpha}_{b}\left(-\frac{5}{12} \tilde{\alpha}_{Y}-\frac{9}{4} \tilde{\alpha}_{2}-8 \tilde{\alpha}_{3}+\frac{1}{2} \tilde{\alpha}_{t}+\frac{9}{2} \tilde{\alpha}_{b}+\tilde{\alpha}_{\tau}\right) \\
\frac{d \tilde{\alpha}_{\tau}}{d t} & =\tilde{\alpha}_{\tau}\left(-\frac{15}{4} \tilde{\alpha}_{Y}-\frac{9}{4} \tilde{\alpha}_{2}+3 \tilde{\alpha}_{b}+\frac{5}{2} \tilde{\alpha}_{\tau}\right) \\
\frac{d v_{u}}{d t} & =\frac{v_{u}}{2}\left(\frac{3}{4} \tilde{\alpha}_{Y}+\frac{9}{4} \tilde{\alpha}_{2}-3 \tilde{\alpha}_{t}\right) \\
\frac{d v_{d}}{d t} & =\frac{v_{d}}{2}\left(\frac{3}{4} \tilde{\alpha}_{Y}+\frac{9}{4} \tilde{\alpha}_{2}-3 \tilde{\alpha}_{b}-\tilde{\alpha}_{\tau}\right)
\end{aligned}
$$

where $t=2 \ln \mu$.

Further, if we define $v^{2}=v_{u}^{2}+v_{d}^{2}$, with $v_{u}=v \sin \beta, v_{d}=v \cos \beta$ and $v \sim 174 \mathrm{GeV}$, the $Z$-boson mass is given by $M_{Z}^{2}=\frac{1}{2}\left(g_{Y}^{2}+g_{2}^{2}\right) v^{2}$. The elecromagnetic and the strong couplings are defined in the usual way

$$
\tilde{\alpha}_{e}=\tilde{\alpha}_{Y} \cos ^{2} \theta_{W}=\tilde{\alpha}_{2} \sin ^{2} \theta_{W}
$$

while the top and bottom quark masses are related to the Higgs vevs by

$$
m_{t}=4 \pi v_{u} \sqrt{\tilde{\alpha}_{t}} \quad m_{b}=4 \pi v_{d} \sqrt{\tilde{\alpha}_{b}}
$$

We will examine the possibility of obtaining $b-\tau$ unification at a low string scale $M_{S}$. We first concentrate in the models (a)-(g) discussed in the previous section. We present our results in the last column of Table 3 .

We notice that $b-\tau$ unification is obtained in model (c), for $M_{S} \approx 2 \times 10^{6} \mathrm{GeV}$. Models (b,e,g) with unification scale $M_{S} \approx 5.8 \times 10^{3} \mathrm{GeV}$ predict a small (25\%) deviation from exact $b-\tau$ unification. We observe that in these cases the strong-weak gauge coupling ratio is $a_{3} \approx 2 a_{2}$. As noted previously, the relation $\alpha_{3}=2 \alpha_{2}$ holds naturally if we embed the model in a $U(3) \times U(2)^{2} \times U(1)^{2}$ symmetry.

In figure 3 the ratio $\frac{m_{b}}{m_{\tau}}$ is plotted as a function of the energy scale for the case of the two-Higgs Standard Model ${ }^{6}$. All previous uncertainties are incorporated and the result is the shaded region shown in the figure. The horizontal shaded band is defined between the values $\frac{m_{b}}{m_{\tau}}=[0.95-1.05]$ taking into account deviations of the ratio $\frac{m_{b}}{m_{\tau}}$ from unity due to possible threshold as well as mixing effects in the full $3 \times 3$ quark and lepton flavor mass 


\begin{tabular}{|c|c|c|c|c|}
\hline model & $k_{Y}$ & $\xi=\frac{\alpha_{2}}{\alpha_{3}}$ & $M_{S} / G e V$ & $\frac{m_{b}}{m_{\tau}}\left(M_{S}\right)$ \\
\hline b,e,g & 2.969 & 0.42 & $5.786 \times 10^{3}$ & 1.25 \\
\hline $\mathrm{c}$ & 2.539 & 0.58 & $1.986 \times 10^{6}$ & 1.01 \\
\hline $\mathrm{d}$ & 1.554 & 0.93 & $1.645 \times 10^{14}$ & 0.73 \\
\hline $\mathrm{a}$ & 1.226 & 1.01 & $6.710 \times 10^{17}$ & 0.68 \\
\hline
\end{tabular}

Table 3: The String scale and the ratio $\xi=\frac{\alpha_{2}}{a_{3}}$ for various orientations of $U(1)$ branes presented in Table 2. The last column shows the $b-\tau$ ratio at $M_{S}$. Exact $b-\tau$-unification is obtained in model (c) for $M_{S} \sim 10^{3} \mathrm{TeV}$.

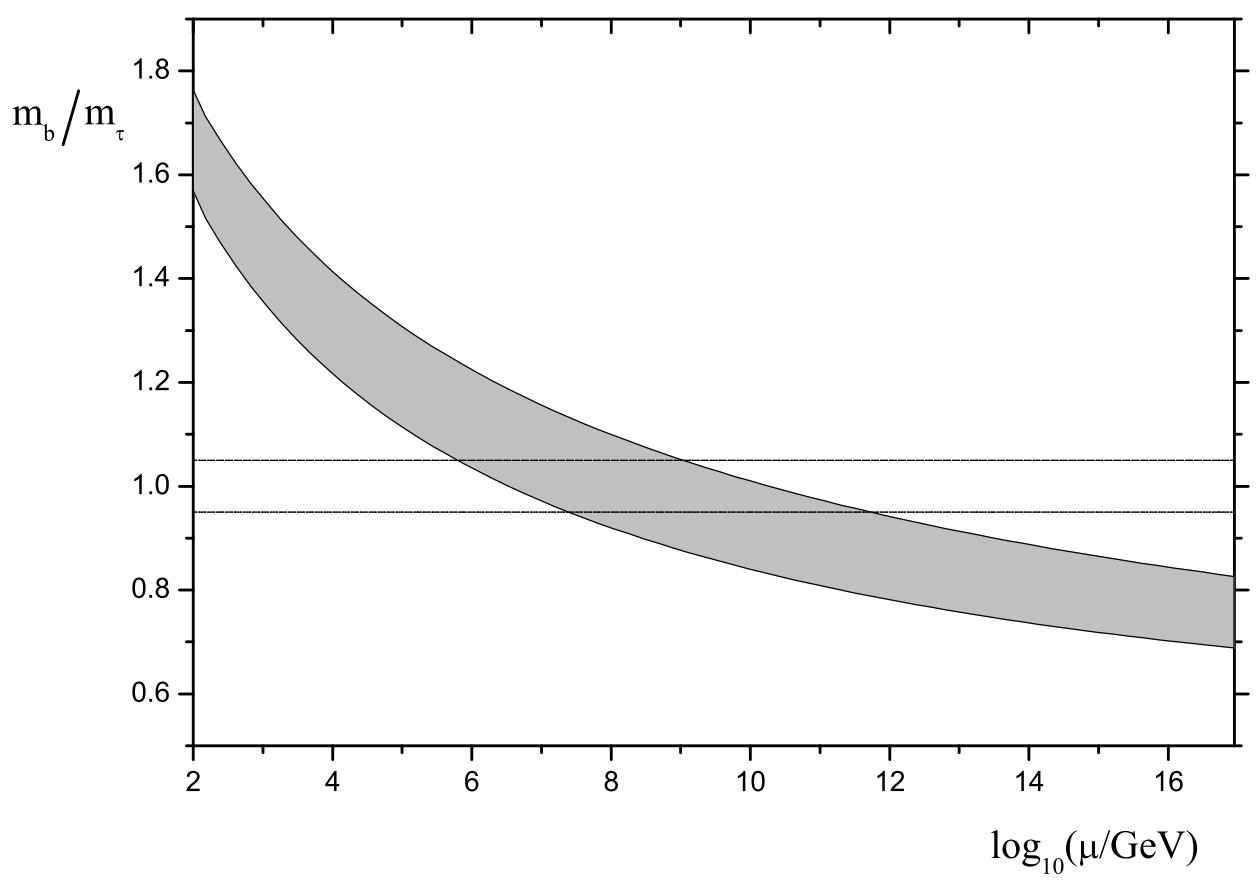

Figure 3: The ratio $\frac{m_{b}}{m_{\tau}}$ as a function of the energy $\mu$ in the 2-Higgs Standard Model. The shaded region corresponds to $a_{3}$ uncertainties. The two horizontal lines indicate the interval $\pm 5 \%$ around the unity.

matrices. As can be seen, exact $m_{b}=m_{\tau}$ equality is found around the scale $M_{S} \approx 2 \times 10^{6}$ $\mathrm{GeV}$. Taking into consideration $m_{b} / m_{\tau}$-uncertainties expressed through the shaded band, the $M_{S}$ energy range is extended up to $\sim 10^{12} \mathrm{GeV}$.

\footnotetext{
${ }^{6}$ For recent work on a 2 Higgs model see [28] and references therein.
} 


\section{Conclusions}

In this letter, we performed a systematic study of the Standard Model embedding in brane configurations with $U(3) \times U(2) \times U(1)^{N}$ gauge symmetry and we examined a number of interesting phenomenological issues. Seeking for models with economical Higgs sector, we identified two brane configurations with two or three $(N=2,3) U(1)$-branes which can accommodate the Standard Model where only one Higgs doublet couples to the up quarks, and a second one couples to the down quarks and leptons. We analysed the possible hypercharge embeddings and found seven possible solutions leading to six models (with acceptable string scale $M_{S}$ ), implying the correct charge assignments for all standard model particles.

We further examined the gauge coupling evolution in these models for both, parallel, as well as intersecting branes and determined the lowest string scale allowed for all possible alignments of the $U(1)$ branes with respect to the $U(3)$ and $U(2)$ non-abelian factors of the gauge symmetry. In the parallel brane scenario, we have identified three models which allow a string scale $M_{S}$ as low as a few TeV, one model with string scale of the order $10^{6}$ $\mathrm{GeV}$ and two models with high unification scales. Similar results were obtained for the general case of intersecting branes.

We further analysed the consequences of the third generation fermion mass relations and in particular $b-\tau$ equality at the string scale on the above models. In the parallel brane scenario, we found that exact $b-\tau$ Yukawa unification is obtained only in the model with $M_{S} \approx 10^{3} \mathrm{TeV}$, while in the $\mathrm{TeV}$ string scale models the $m_{b} / m_{\tau}$ ratio deviates from unity by $25 \%$. Allowing the $U(1)$ gauge couplings to take arbitrary (perturbative) values, we found that $b-\tau$ Yukawa unification is possible for a wide string scale range form $10^{6}$ up to $10^{12} \mathrm{GeV}$.

Acknowledgements. This research was funded by the program 'PYTHAGORAS' (no. 1705 project 23) of the Operational Program for Education and Initial Vocational Training of the Hellenic Ministry of Education under the 3rd Community Support Framework and the European Social Fund. GKL and JR would like to acknowledge kind hospitality of Ecole Normale Superiere in Paris, where part of this work has been done. 


\section{References}

[1] I. Antoniadis, Phys. Lett. B 246 (1990) 377.

[2] N. Arkani-Hamed, S. Dimopoulos and G. R. Dvali, Phys. Lett. B 429 (1998) 263 arXiv:hep-ph/9803315.

[3] I. Antoniadis, N. Arkani-Hamed, S. Dimopoulos and G. R. Dvali, Phys. Lett. B 436 (1998) 257 arXiv:hep-ph/9804398.

[4] J. D. Lykken, Phys. Rev. D 54 (1996) 3693 arXiv:hep-th/9603133.

[5] J. Polchinski, arXiv:hep-th/9611050.

[6] C. Angelantonj and A. Sagnotti, Phys. Rept. 371 (2002) 1 [Erratum-ibid. 376 (2003) 339] arXiv:hep-th/0204089.

[7] M. Berkooz, M. R. Douglas and R. G. Leigh, Nucl. Phys. B 480 (1996) 265 arXiv:hep-th/9606139.

[8] V. Balasubramanian and R. G. Leigh, Phys. Rev. D 55 (1997) 6415 arXiv:hep-th/9611165.

[9] I. Antoniadis, E. Kiritsis and T. N. Tomaras, Phys. Lett. B 486 (2000) 186 hep-ph/0004214.

[10] I. Antoniadis, E. Kiritsis, J. Rizos and T. N. Tomaras, Nucl. Phys. B 660 (2003) 81 arXiv:hep-th/0210263.

[11] I. Antoniadis, E. Kiritsis and J. Rizos, Nucl. Phys. B 637 (2002) 92 arXiv:hep-th/0204153.

[12] G. Aldazabal, S. Franco, L. E. Ibanez, R. Rabadan and A. M. Uranga, JHEP 0102 (2001) 047 arXiv:hep-ph/0011132. ; L. E. Ibanez, F. Marchesano and R. Rabadan, JHEP 0111 (2001) 002 arXiv:hep-th/0105155. ; R. Blumenhagen, B. Kors, D. Lust and T. Ott, Nucl. Phys. B 616 (2001) 3 arXiv:hep-th/0107138.

[13] M. Cvetic, G. Shiu and A. M. Uranga, Phys. Rev. Lett. 87 (2001) 201801 arXiv:hep-th/0107143. ; M. Cvetic, G. Shiu and A. M. Uranga, Nucl. Phys. B 615 (2001) 3 |arXiv:hep-th/0107166; ; R. Blumenhagen, M. Cvetic, P. Langacker and G. Shiu, arXiv:hep-th/0502005.

[14] I. Antoniadis, E. Kiritsis and J. Rizos, Nucl. Phys. B 637 (2002) 92 arXiv:hep-th/0204153.

[15] G. K. Leontaris and J. Rizos, Phys. Lett. B 510 (2001) 295 hep-ph/0012255. 
[16] C. Kokorelis, JHEP 0208 (2002) 018 arXiv:hep-th/0203187. ; C. Kokorelis, Nucl. Phys. B 677 (2004) 115 arXiv:hep-th/0207234. ; L. L. Everett, G. L. Kane, S. F. King, S. Rigolin and L. T. Wang, Phys. Lett. B 531 (2002) 263 arXiv:hep-ph/0202100. ; G. C. Branco, J. M. Gerard, F. R. Gonzalez and B. M. Nobre, arXiv:hep-ph/0305092; M. Axenides, E. Floratos and C. Kokorelis, JHEP 0310 (2003) 006 arXiv:hep-th/0307255.

[17] R. Blumenhagen, D. Lust and S. Stieberger, JHEP 0307 (2003) 036 arXiv:hep-th/0305146.

R. Blumenhagen, M. Cvetic, F. Marchesano and G. Shiu, JHEP 0503 (2005) 050 arXiv:hep-th/0502095.

[18] I. Antoniadis and S. Dimopoulos, Nucl. Phys. B 715 (2005) 120 arXiv:hep-th/0411032.

[19] R. Blumenhagen, M. Cvetic, P. Langacker and G. Shiu, arXiv:hep-th/0502005, D. Cremades, L. E. Ibanez and F. Marchesano, JHEP 0405 (2004) 079 arXiv:hep-th/0404229; P. Anastasopoulos, arXiv:hep-th/0503055.

[20] V. S. Kaplunovsky, Nucl. Phys. B 307 (1988) 145 [Erratum-ibid. B 382 (1992) 436] arXiv:hep-th/9205068. I. Antoniadis, K. S. Narain and T. R. Taylor, Phys. Lett. B 267 (1991) 37. H. P. Nilles and S. Stieberger, Nucl. Phys. B 499 (1997) 3 arXiv:hep-th/9702110. P. M. Petropoulos and J. Rizos, Phys. Lett. B 374 (1996) 49 arXiv:hep-th/9601037.

[21] M. S. Chanowitz, J. R. Ellis and M. K. Gaillard, Nucl. Phys. B 128 (1977) 506. A. J. Buras, J. R. Ellis, M. K. Gaillard and D. V. Nanopoulos, Nucl. Phys. B 135 (1978) 66. B. Ananthanarayan, G. Lazarides and Q. Shafi, Phys. Rev. D 44 (1991) 1613. M. Carena, M. Olechowski, S. Pokorski and C. E. M. Wagner, Nucl. Phys. B 426 (1994) 269 arXiv:hep-ph/9402253.

[22] B. R. Greene, K. H. Kirklin, P. J. Miron and G. G. Ross, Nucl. Phys. B 292 (1987) 606. I. Antoniadis and G. K. Leontaris, Phys. Lett. B 216 (1989) 333. I. Antoniadis, G. K. Leontaris and J. Rizos, Phys. Lett. B 245 (1990) 161. A. E. Faraggi, Phys. Lett. B 274 (1992) 47. G. Cleaver, M. Cvetic, J. R. Espinosa, L. L. Everett, P. Langacker and J. Wang, Phys. Rev. D 59 (1999) 115003 arXiv:hep-ph/9811355. G. K. Leontaris and J. Rizos, Nucl. Phys. B 554 (1999) 3 arXiv:hep-th/9901098.

[23] E. Witten, Nucl. Phys. B 471 (1996) 135 arXiv:hep-th/9602070.

[24] E. Kiritsis, Fortsch. Phys. 52 (2004) 200 arXiv:hep-th/0310001, and references therein.

[25] S. Eidelman et al., Phys. Lett. B592, 1 (2004)

[26] M. K. Parida and A. Usmani, Phys. Rev. D 54 (1996) 3663.

C. R. Das and M. K. Parida, Eur. Phys. J. C 20 (2001) 121 arXiv:hep-ph/0010004. 
[27] H. Arason, D. J. Castano, B. Keszthelyi, S. Mikaelian, E. J. Piard, P. Ramond and B. D. Wright, Phys. Rev. D 46 (1992) 3945.

[28] G. L. Kane, S. F. King, I. N. R. Peddie and L. Velasco-Sevilla, arXiv:hep-ph/0504038. 